\title{
Simulations of GRB Jets in a Stratified External
} Medium

\author{
Fabio De Colle* \\ TASC, Department of Astronomy and Astrophysics, University of California, Santa Cruz, CA \\ 95064, USA \\ E-mail: fabioducolick.org
}

\begin{abstract}
We present multi-dimensional simulations of GRB jets propagating both in uniform and stratified external media. The simulations are performed in two dimensions using a newly developed, special relativistic, adaptive mesh refinement hydrodynamics code. We describe the differences in the dynamics of the GRB jet as a function of the stratification of the ambient medium and, by post-processing the results of the simulations with a radiation code, we compute the afterglow emission from the GRB as it decelerates from highly relativistic to Newtonian velocities.
\end{abstract}

Gamma-Ray Bursts 2012 Conference -GRB2012,

May 07-11, 2012

Munich, Germany

${ }^{*}$ Speaker. 


\section{Introduction}

The dynamics of a Gamma-Ray Burst (GRB) can be broadly divided in three phases. When $u=\Gamma v / c \gtrsim 1 / \theta_{0}$ (where $u$ is the velocity quadrivector, $\Gamma$ is the Lorentz factor of the GRB jet, $\theta_{0}$ is its opening angle and $v$ is its velocity), the dynamics of the system can be described by the self-similar Blandford \& McKee (1976) solution. On much larger timescales, when $u \ll 1$ and the flow is, in addition to being non-relativistic, also spherical, the Sedov-Taylor self-similar solution applies. In the intermediate cases $(u \approx 1)$, there are not analytical solutions, and numerical simulations must be employed.

Numerical studies have been until recently limited to the case of a uniform medium. Actually, the association of GRBs with type Ic supernovae implies that the GRB will move in a medium shaped by the propagation of massive Wolf-Rayet winds (although modeling of GRB observations seems to favor a uniform density distribution).

We shortly describe in this presentation the results of our recent study of the dynamics of GRBs as they move through a stratified environment (De Colle et al. 2012b), showing that the deceleration and lateral expansion of the relativistic flow as it becomes non-relativistic strongly depend on the density distribution of the ambient medium.

\section{Methods}

To compute the dynamics of decelerating GRBs, we use the code Mezcal, a finite-volume, conservative, adaptive mesh refinement code, used to integrate hyperbolic equations. In its current version, it includes hydrodynamics and magnetohydrodynamics, both in their Newtonian and special relativistic form. Extra physics modules include cooling, thermal conduction, external gravity, resistivity, and nuclear reaction. The code is currently used to study a relatively broad range of astrophysical problems, i.e. supernova remnants, GRBs, Herbig-Haro jets, or planet-magnetospheric interaction, among others (see the figure below).

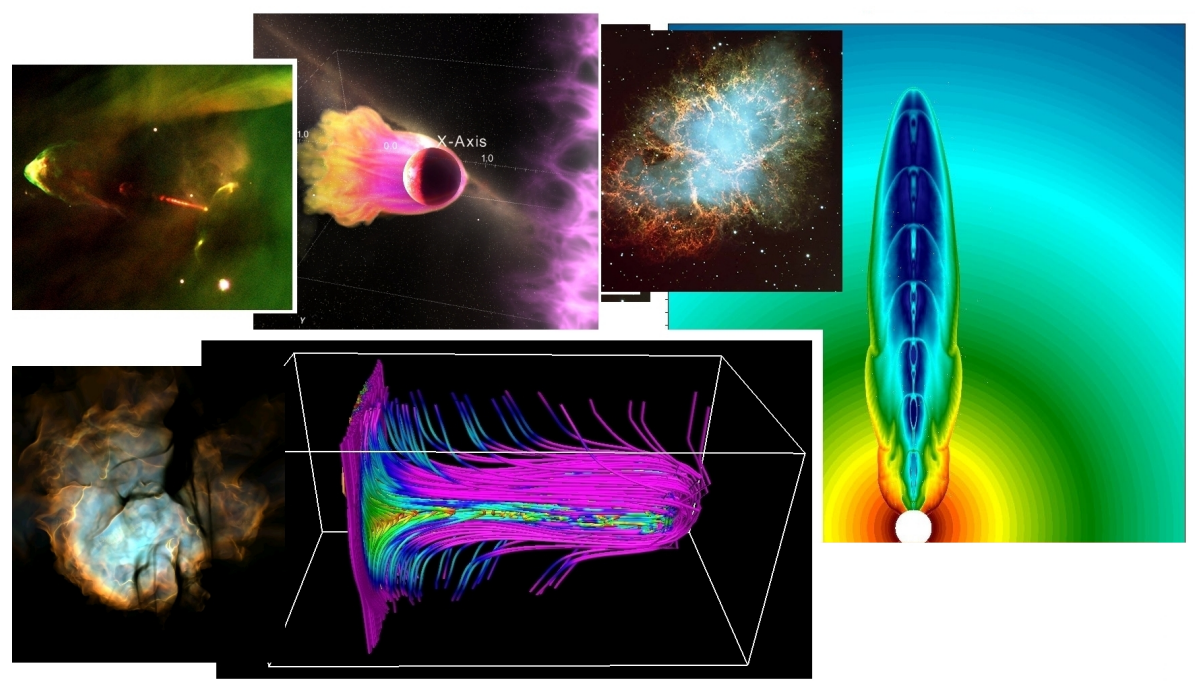


The afterglow radiation is computed by assuming standard synchrotron radiation. The microphysics of the acceleration and emission processes is parameterized by taking the "standard" parameters $\varepsilon_{e}=\varepsilon_{B}=0.1$ and $p=2.5$. In our simple prescription for electron cooling, which is similar to the one used by Granot et al. (2001) and Zhang \& MacFadyen (2009), the electrons are assumed to have cooled at their current local cooling rate over the dynamical time

\section{Results: 1D}

The Mezcal code has been used (see De Colle et al. 2012a) to study the propagation of onedimensional (1D) spherical impulsive blast waves expanding in a stratified medium with $\rho \propto r^{-k}$, bridging between the relativistic and Newtonian phases (which are described by the BlandfordMcKee and Sedov-Taylor self-similar solutions, respectively).

The evolution of the shock front radius as a function of time for the three cases $k=0,1,2$ (up to bottom) along with the ultra-relativistic $\left(R_{s h}=c t\right)$ and the Sedov-Taylor $\left(R_{\mathrm{sh}}=\left(\alpha_{k} E_{\mathrm{iso}} t^{2} / A_{k}\right)^{1 /(5-k)}\right)$ regimes is shown in the following Figure.

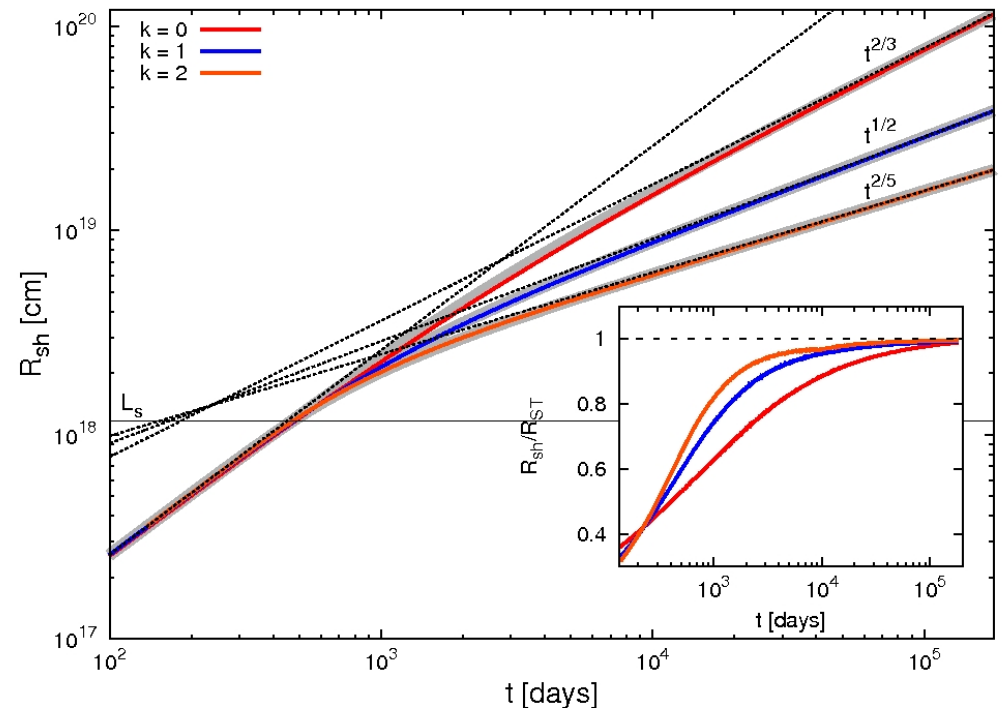

In the figure, the gray thick curves are computed from a simple semi-analytical approximation based on the following argument. As the energy is in general given by $E \propto R^{3-k} \beta^{2} \gamma^{2}$, we take a simple interpolation between the ultra-relativistic and non-relativistic limits by

$$
E=R^{3-k} \beta^{2} \Gamma^{2} A_{k} c^{2}\left(\frac{8 \pi}{17-4 k} \beta^{2}+\frac{(5-k)^{2}}{4 \alpha_{k}}\left(1-\beta^{2}\right)\right)
$$

This equation can be easily written as function of velocity as:

$$
\beta^{2}=\frac{2}{1+c_{N R}\left(R / L_{s}\right)^{3-k}+\sqrt{\left[1-c_{\mathrm{NR}}\left(R / L_{\mathrm{s}}\right)^{3-k}\right]^{2}+4 c_{R}\left(R / L_{\mathrm{s}}\right)^{3-k}}}
$$

where $c_{R}=\frac{2(3-k)}{17-4 k}$ and $c_{\mathrm{NR}}=\frac{(5-k)^{2}(3-k)}{16 \pi \alpha_{k}}$, and can be easily integrated numerically to give the shock radius as a function of time. The resulting curve approximates the numerical solution within a few percent. 
The 1D simulations show that the deceleration to non-relativistic speeds in one-dimension occurs on scales significantly larger than the Sedov length. This transition is further delayed with respect to the Sedov length as the degree of stratification of the ambient medium is increased.

\section{Results: 2D}

We initialize our GRB jet in 2D as a conical wedge of half-opening angle $\theta_{0}$, within which the initial radial profiles of pressure, density and Lorentz factor in the post-shock region are taken from the spherical Blandford-McKee self-similar solutions for a stratified medium. Two-dimensional simulations with $k=0$ (homogeneous medium), $k=1$ and $k=2$ (corresponding to a steady stellar wind medium) are then evolved to study the lateral expansion and deceleration of the jet.

The figure below (taken from De Colle et al. 2012b) shows the evolution of the GRB jets as a function of time (increasing from left to right) and stratification (with $k=0,1,2$ from top to bottom).

At the beginning of the simulation, there is a transient phase with the formation of an slowly expanding egg-like structure. The expansion velocity remains mainly radial at small angles and non-relativistic (and in the $\theta$ direction) at large angles. In addition, the lateral expansion increases with $k$. In brief, the jet mass is mostly located at the edge of the jet (at large $\theta$ values) but most of the energy remain confined near the head of the jet. The early expansion is faster with increasing values of $k$, while the later expansion is slower. Therefore, GRBs moving in more stratified media will achieve spherical symmetry on a larger timescale.

\section{Conclusions}

The jet dynamics from our 2D simulations for the case with $k=0$ and the resulting afterglow lightcurves, including the jet break, are in good agreement with those presented in previous works (e.g. Granot et al. 2001, Zhang \& MacFadyen 2009).

However, the dynamics of the GRB jets is greatly modified by the density stratification of the environment where the GRB decelerates. In particular, for a more stratified medium (e.g. $k=2$ vs $k=0$ ): faster (slower) jet lateral expansion is achieved at early (late) times; the jet break is smoother due to the density stratification; the counter-jet contribution remains smaller, possibly explaining the lack of observed counter-jets.

\section{References}

[1] F. De Colle, et al., Gamma-Ray Burst Dynamics and Afterglow Radiation from Adaptive Mesh Refinement, Special Relativistic Hydrodynamic Simulations, ApJ, 751 (2012) 57 [astro-ph/1111.6890]

[2] F. De Colle, et al., Simulations of Gamma-Ray Burst Jets in a Stratified External Medium: Dynamics, Afterglow Light Curves, Jet Breaks, and Radio Calorimetry, ApJ, 746 (2012) 122 [astro-ph/1111.6667]

[3] R.D. Blandford \& C.F. McKee, Fluid Dynamics of Relativistic blast waves, ApJ, 19 (1976) 1130 

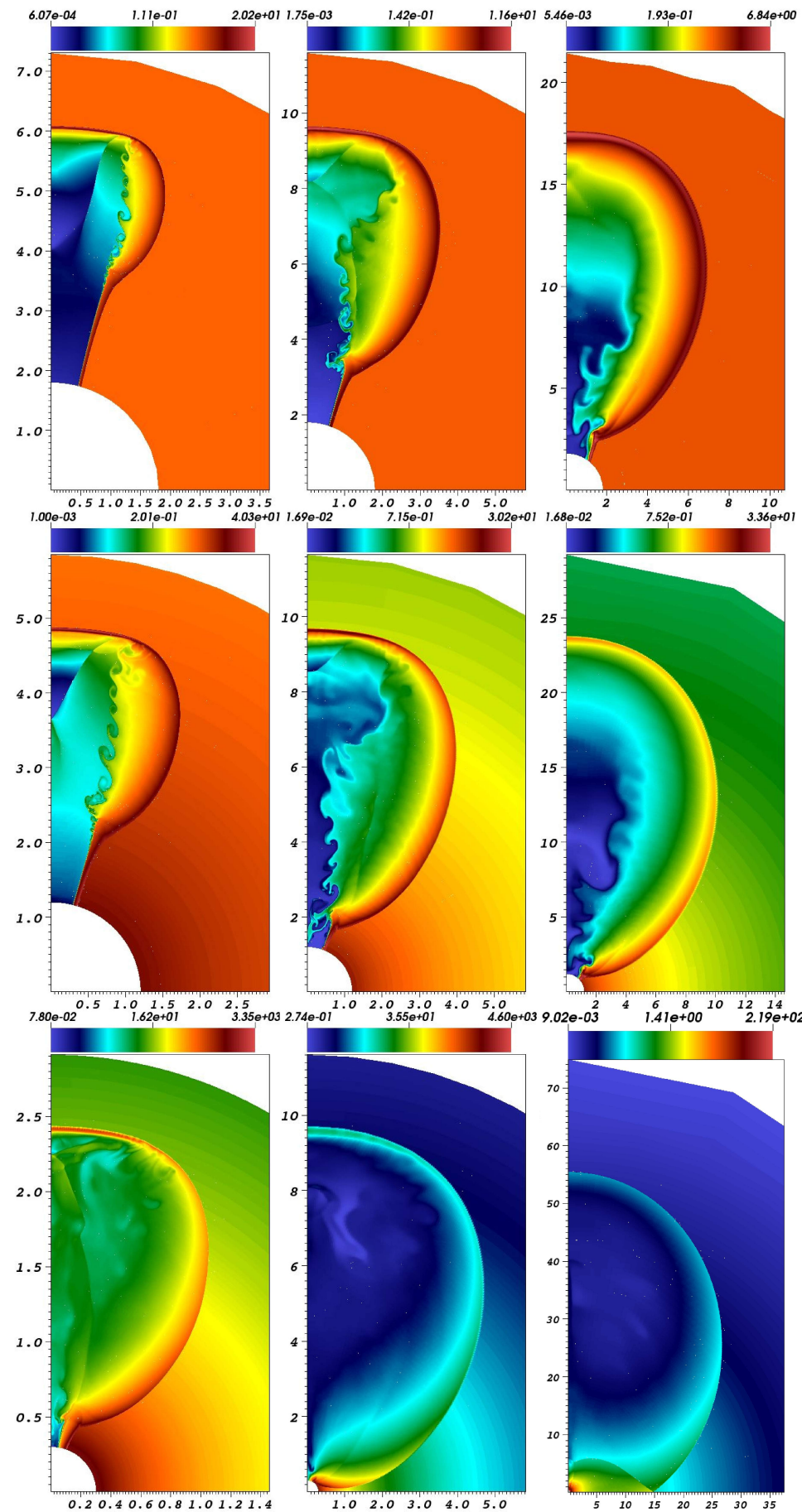
[4] J. Granot, et al., Light curves from an expanding relativistic jet, in proceedings of Gamma-Ray Bursts in the Afterglow Era, Springer-Verlag 312 (2001) [astro-ph/ 0103038 ]

[5] W. Zhang \& A. MacFadyen The Dynamics and Afterglow Radiation of Gamma-Ray Bursts. I. Constant Density Medium, ApJ, 698 (2009) 1261 [astro-ph/ 0902 . 2396] 